\title{
QGSJET-II: results for extensive air showers
}

\author{
S. Ostapchenko ${ }^{a b * \dagger}$ \\ anstitut für Experimentelle Kernphysik, University of Karlsruhe, 76021 Karlsruhe, Germany \\ ${ }^{b}$ D.V. Skobeltsyn Institute of Nuclear Physics, Moscow State University, 119992 Moscow, Russia
}

The new hadronic Monte Carlo model QGSJET-II is applied for extensive air shower (EAS) calculations. The obtained results are compared to the predictions of the original QGSJET and of the SIBYLL 2.1 interaction models. It is shown that non-linear effects change substantially model predictions for hadron-nucleus interactions and produce observable effects for calculated EAS characteristics. Finally the impact of the new model on the interpretation of air shower array data is discussed.

\section{INTRODUCTION}

Experimental studies of high energy cosmic rays $(\mathrm{CR})$ are mainly performed on the basis of extensive air shower (EAS) techniques: measuring various characteristics of nuclear-electromagnetic cascades, induced by primary CR particles in the atmosphere, one uses the obtained knowledge to reconstruct the properties of the initial particles. Because of a very complicated structure of the atmospheric cascade such an analysis necessarily requires both particle detectors - to measure various EAS components at Earth or in space, and proper simulation tools, which allow to mimic the cascade process and to establish a connection between the measured information and the primary particle characteristics. An important part of EAS simulation programs are Monte Carlo (MC) models of hadronic interactions. In the absence of a rigorous theoretical approach for a treatment of general (minimum-bias) hadron-nucleus and nucleus-nucleus collisions a development of reliable hadronic MC generators for very high energy interactions is far non-trivial; also due to the necessity to extrapolate corresponding experimental knowledge from the energies of present

\footnotetext{
*Now at Forschungszentrum Karlsruhe, Institut für Kernphysik, 76021 Karlsruhe, Germany.

${ }^{\dagger}$ This work has been supported in part by the German Ministry for Education and Research (BMBF, Grant 05 CU1VK1/9).
}

colliders to much higher CR energies. On the other hand, measuring different EAS components allows to perform a cross-check of model description and to discriminate between available MC generators.

During last years QGSJET model [123] has been successfully used in the field, in particular, being employed in popular EAS simulation programs, CORSIKA [4] and AIRES [5]. Also model tests, performed for example by the KASCADE collaboration, using its multi-component EAS detector setup [6], showed that the model reproduces measured shower characteristics and their correlations reasonably well 78 . However, the mentioned agreement is still far from being ideal; recent investigations showed some discrepancies in the description of basic correlations between electron and muon EAS components 9]. Also annoying is the fact that analyzing different EAS components results in sizably divergent conclusions concerning primary CR composition, for all models in use 1011 .

Recently a new hadronic MC model QGSJETII has been developed [12, which included a treatment of non-linear interaction effects in individual hadronic (nuclear) collisions. The latter gave a possibility to obtain a consistent description of interaction cross sections and parton momentum distributions in hadrons, compared to corresponding measurements, thus solving the basic deficiency of the original QGSJET and providing 
a much more reliable model extrapolation into ultra-high energy domain. Additionally, realistic nuclear density parameterizations have been employed in the model, individually for each nucleus [13, more reliable two-component low mass diffraction treatment has been used [1, and all the model parameters have been re-calibrated using a wider set of accelerator data. The goal of the present work is to investigate the impact of the mentioned modifications on the calculated air shower characteristics and to draw possible consequences for EAS data interpretation.

\section{RESULTS FOR HADRON-NUCLEUS INTERACTIONS}

Despite the fact that the physics of hadronic interactions is very complicated and contributes to air shower development in a rather non-trivial way, basic EAS observables mainly depend on a limited number of macroscopic characteristics of hadron-air collisions. Indeed, the most fundamental EAS parameter, the position of the shower maximum $X_{\max }$ is grossly defined by inelastic hadron-air cross sections and by the interaction inelasticities, the latter quantity being defined as the relative difference between the lab. energies of the initial and the most energetic final particles. While the number of charged leptons $N_{e}$, measured at a given observation level, is strongly correlated with $X_{\max }$, this correlation is much weaker for corresponding number of muons $N_{\mu}$, and even becomes negative for relatively high muon threshold. On the other hand, muon numbers appear to be quite sensitive to multiplicities of elementary interactions.

In Figs. 10 3 the predictions of the new model for inelastic cross sections $\sigma_{h-\text { air }}^{\text {inel }}$, inelasticities $K_{h \text {-air }}^{\text {inel }}$, and multiplicities of charged particles $N_{h \text {-air }}^{\text {ch }}$ are plotted in comparison with both the original QGSJET and the SIBYLL 2.1 [14 models. Compared to the original model, the results of the QGSJET-II are defined by a competition of two effects: non-linear screening corrections, which lead to a reduction of the interaction eikonal and correspondingly of the number of elementary particle production processes (cut Pomerons), and steeper parton momentum dis-

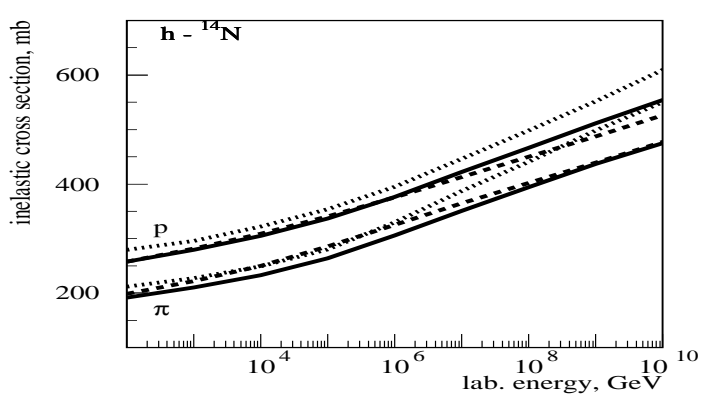

Figure 1. Inelastic hadron-air cross sections for QGSJET-II, QGSJET-I, and SIBYLL 2.1 models - smooth, dashed, and dotted curves.

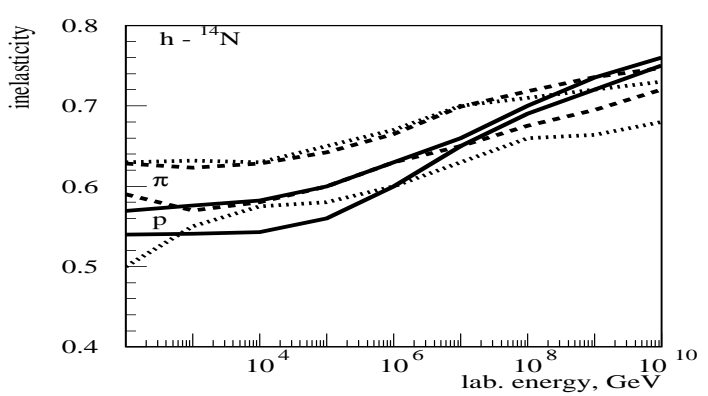

Figure 2. Inelasticities of hadron-air interactions for QGSJET-II, QGSJET-I, and SIBYLL 2.1 models - smooth, dashed, and dotted curves.

tributions, leading to a faster energy increase of the latter quantities 12. At not too high energies the first effect dominates, leading to smaller numbers of $\sigma_{h-\text { air }}^{\text {inel }}, K_{h \text {-air }}^{\text {inel }}$, and $N_{h \text {-air }}^{\text {ch }}$, especially for pion-air interactions. On the other hand, in the very high energy limit the influence of parton distributions prevails and the new model predicts larger values for the quantities of interest.

\section{AIR SHOWER CHARACTERISTICS}

Sizably smaller inelasticities of the new model lead to a somewhat deeper position of the shower maximum, compared to the original QGSJET Fig. 4 The corresponding shift of $X_{\max }$ changes from about $20 \mathrm{~g} / \mathrm{cm}^{2}$ at $10^{14} \mathrm{eV}$ to $\sim 10 \mathrm{~g} / \mathrm{cm}^{2}$ at $10^{20} \mathrm{eV}$ in case of proton-induced EAS; for ironinduced EAS it increases slowly from $15 \mathrm{~g} / \mathrm{cm}^{2}$ till $\sim 20 \mathrm{~g} / \mathrm{cm}^{2}$. As the relative strength of non-linear effects is larger for nucleus-nucleus collisions one 


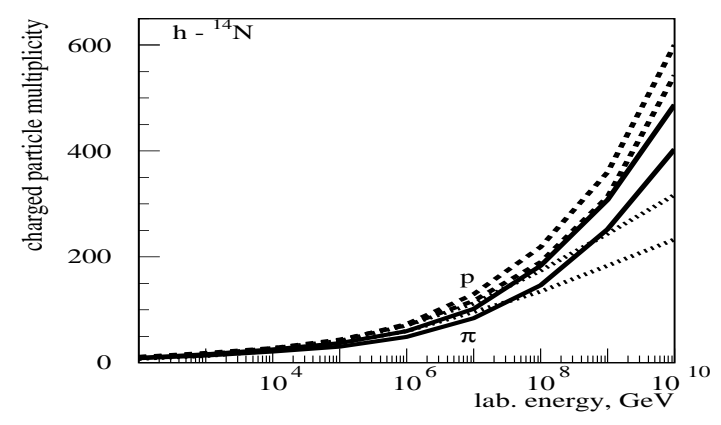

Figure 3. Multiplicities of charged particles in hadron-air interactions for QGSJET-II, QGSJET-I, and SIBYLL 2.1 models - smooth, dashed, and dotted curves.

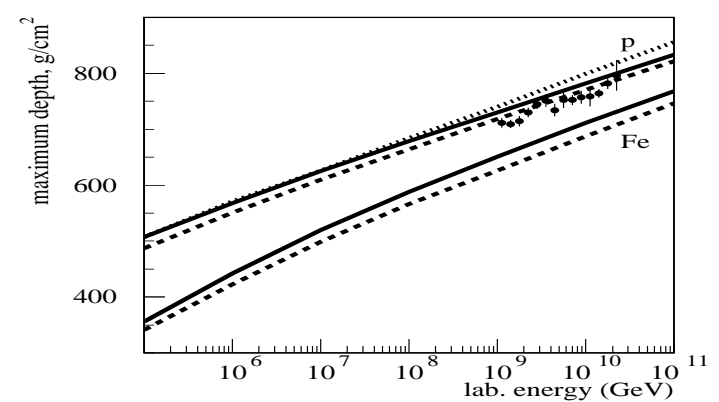

Figure 4. Average position of the shower maximum for proton- and iron-induced EAS for QGSJET-II, QGSJET-I, and SIBYLL 2.1 models - smooth, dashed, and dotted curves. The points are the data of the HIRES collaboration [15].

observes sizable deviations from the superposition picture for nucleus-induced air showers.

The relative difference between QGSJET-II and QGSJET-I models for predicted numbers of electrons and muons $\left(E_{\mu}>10 \mathrm{GeV}\right)$ at sea level for vertical proton- and iron-induced showers is shown in Figs. 5 6] for comparison the $N_{\mu}$ difference between SIBYLL 2.1 and QGSJET-I is also shown for the case of primary protons. While for electron numbers we observe the expected correlation with the position of the shower maximum, the predicted muon numbers in the new model are significantly smaller, by as much as $30 \%$ at highest energies, due to a strong reduction of interaction multiplicity, especially for pion-nucleus

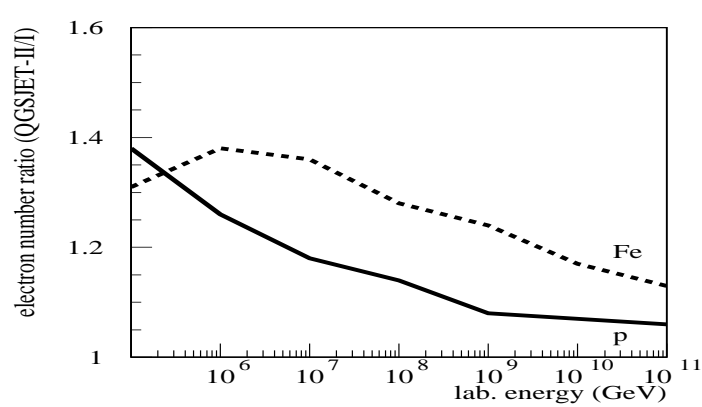

Figure 5. Relative $N_{e}$-difference at sea level between QGSJET-II and QGSJET-I models for vertical proton- and iron-induced EAS - smooth and dashed curves.

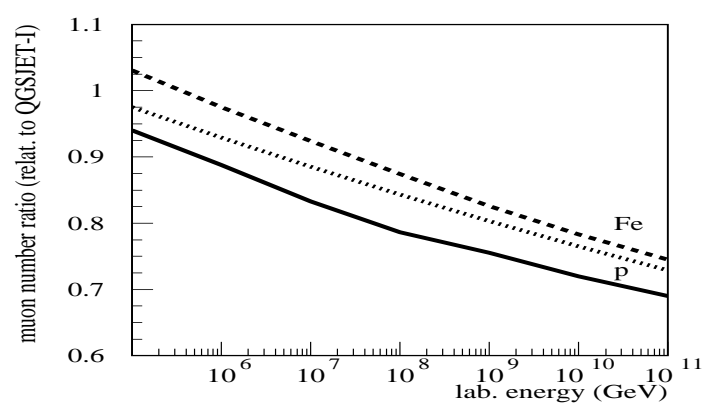

Figure 6. Relative $N_{\mu}$-difference $\left(E_{\mu}>10 \mathrm{GeV}\right)$ at sea level between QGSJET-II and QGSJETI models for vertical proton- and iron-induced EAS - smooth and dashed curves, and between SIBYLL 2.1 and QGSJET-I models for $p$-induced EAS - dotted curve.

interactions, with the results being close to the predictions of the SIBYLL 2.1 model.

\section{DISCUSSION}

Systematic account for non-linear screening effects in the QGSJET-II model results in a substantial reduction of multiplicity and inelasticity of hadron-nucleus interactions compared to the original QGSJET. This leads in turn to a shift of the predicted position of the shower maximum deeper in the atmosphere, to larger number of electrons and smaller number of muons at sea level. Applying the new model to EAS data reconstruction will change present conclu- 
sions concerning CR composition towards heavier primaries. The corresponding $X_{\max }$ change is not large at highest energies. Nevertheless, while the predictions of the original QGSJET, being compared to HIRES measurements, are marginally consistent with the assumption of ultra high energy cosmic rays being only protons, this no longer the case with the new model.

Similarly, at the knee energies QGSJET-II predicts a substantially smaller number of muons for a given number of electrons, which would lead to a conclusion about a sizably heavier composition, compared to what is currently obtained with QGSJET-I. In particular, the mentioned change should bring closer together the composition results of the KASCADE collaboration obtained on the basis of electron/muon and hadronic EAS components [10]. Also the obtained changes seem to go in the right direction to resolve the reported discrepancies in the electron/muon correlations 9. It should be mentioned, however, that at highest energies the disagreement of the composition results obtained with fluorescence light-based measurements and with ground arrays 11 would increase using the new model, as the reduction of EAS muon number at highest energies is much stronger compared to the corresponding effect for the shower maximum.

It is of interest to investigate the impact of the new model on the experimental techniques of primary energy reconstruction, where two methods are mainly used: either based on the integral of the shower cascade curve in case of fluorescence light-based measurements or estimating the energy from charged particle densities at distances 600 and $1000 \mathrm{~m}$ from the shower core [16. In the first case model dependence of the results enters mainly via predicted numbers of charged particles in the shower maximum $N_{e}^{\max }$, which shows very small model sensitivity - less than $3 \%$ between QGSJET-II, QGSJET-I, and SIBYLL 2.1. Similarly, for electron density at large distances from the core one observes just a few per cent shift between the new and the old versions of QGSJET. However, depending on experimental techniques applied, a substantial contribution to the measured signal may come from the muon component, which is the case for example, for AUGER collaboration 17. As the latter is substantially reduced in QGSJET-II, corresponding energy estimates of the ground array would move upwards compared to QGSJET-I. On the other hand, the possibility to perform independent energy reconstructions with both the fluorescence detector and the ground array could allow to perform a model consistency check at the highest CR energies.

\section{REFERENCES}

1. N.N. Kalmykov and S.S. Ostapchenko, Phys. Atom. Nucl. 56 (1993) 346.

2. N.N. Kalmykov et al., Bull. Russ. Acad. Sci. Phys. 58 (1994) 1966.

3. N.N. Kalmykov et al., Nucl. Phys. Proc. Suppl. 52B (1997) 17.

4. D. Heck et al., "CORSIKA: A Monte Carlo code to simulate extensive air showers", Forschungszentrum Karlsruhe, Report FZKA 6019, 1998.

5. S.J. Sciutto, "AIRES: A system for air shower simulations (version 2.2.0)", astro-ph/9911331.

6. T. Antoni et al., KASCADE Collaboration, Nucl. Instr. Meth. A513 (2003) 490.

7. T. Antoni et al., KASCADE collaboration, J. Phys. G: Nucl. Part. Phys. 25 (1999) 2161.

8. J. Milke et al., KASCADE collaboration, Acta Phys. Polon. B35 (2004) 341.

9. H. Ulrich et al., KASCADE collaboration, Proc. 19th Eur. Cosmic Ray Symp. (Florence); Int. J. Mod. Phys. A, to be published.

10. J. Horandel et al., KASCADE collaboration, Nucl. Phys. Proc. Suppl. 110 (2002) 453.

11. A.A. Watson, astro-ph/0410514

12. S. Ostapchenko, Nucl. Phys. Proc. Suppl. B (2005) to be published, hep-ph/0412332.

13. N.N. Kalmykov et al., Proc. $26^{\text {th }}$ Int. Cosmic Ray Conf. (Salt Lake City), v.1, p.419 (1999).

14. R. Engel et al., Proc. $26^{\text {th }}$ Int. Cosmic Ray Conf. (Salt Lake City), v.1, p.415 (1999).

15. R.U. Abbasi et al., HIRES Collaboration, astro-ph/0407622

16. M. Nagano and A.A. Watson, Rev. Mod. Phys. 72 (2000) 689.

17. J. Abraham et al., AUGER Collaboration, Nucl. Instr. Meth. A523 (2004) 50. 